\title{
ANALYSIS OF THE MAGNITUDE-REDSHIFT RELATION INCLUDING POSSIBLE EFFECTS OF EVOLUTION
}

\author{
JAN-ERIK SOLHEIM \\ Institute of Theoretical Astrophysics, Oslo, Norway* \\ and \\ BEATRICE M. TINSLEY \\ University of Texas, Dallas, Texas, U.S.A.
}

\begin{abstract}
In this paper, we compare the magnitude-redshift data for brightest cluster galaxies with cosmological models of general relativity, including non-zero cosmological constant. Our study is parallel to that by Peach (1970). We use the same data but an independent method, and obtain somewhat different results. We also consider possible effects of evolution.
\end{abstract}

The data used in this test are photoelectric $V$-magnitudes from unpublished photometry of Sandage, given indirectly by Peach (1969). The data contain magnitudes corrected for $K$-correction and absorption, for the brightest members of 38 clusters. Of these, ten are radio galaxies. The sample corresponds to $(\mathrm{I}+\mathrm{II}) V_{c}$ in Peach's work. For the $K$-correction we have used two different versions: from Oke and Sandage (1968) and from Whitford (1971); the latter gives better representation of the outer parts of the galaxy.

The procedure used in the tests here was previously described by Solheim (1966). It determines the best model from calculations of the least-square errors of a number of models, given $q_{0}, \sigma_{0}$, and $H_{0}$. With this method, confidence levels can be given as closed curves in the $\left(q_{0}, \sigma_{0}\right)$ model diagram.

Figure 1 shows the result of a test with this data and no correction for evolution, giving the $64 \%$ confidence limits. The best fit is the model with $q_{0}$ about 2 and a negative value for the density, results that are similar to those of Peach (1970).

From this diagram it is seen that we can say very little about the value of $\sigma_{0}$. This is because we only have objects with $z<0.2$ included, for which good photoelectric photometry exists. However, if we look at the only realistic part of the model diagram, with positive densities, we find that models with $\sigma_{0}<4.5$ and $-1.5<q_{0}<1.5$ are acceptable, according to the results of the test with Whitford's $K$-correction.

In this situation, it is possible to choose an acceptably low value of the density parameter $\sigma_{0}$. If we choose the most widely accepted density of $10^{-30} \mathrm{~g} \mathrm{~cm}^{-3}$, this corresponds to $\sigma_{0}=0.05$ (using $H_{0}=75$ ); the rest of this discussion will be based on these values. We then find that the Whitford $K$-correction leads to $q_{0}=0.29 \pm 1.1$ as the best model. Within this range we have oscillating models for $q_{0}>0.05$ and monotonic expanding models for $q_{0}<0.05$ (Stabell and Refsdal, 1966). The age of the best model is $10.8 \times 10^{9} \mathrm{yr}$ for $H_{0}=75$.

Tinsley (1970) has recently proposed a model for evolution of giant elliptical

* Present address: University of Tromsö, Norway. 


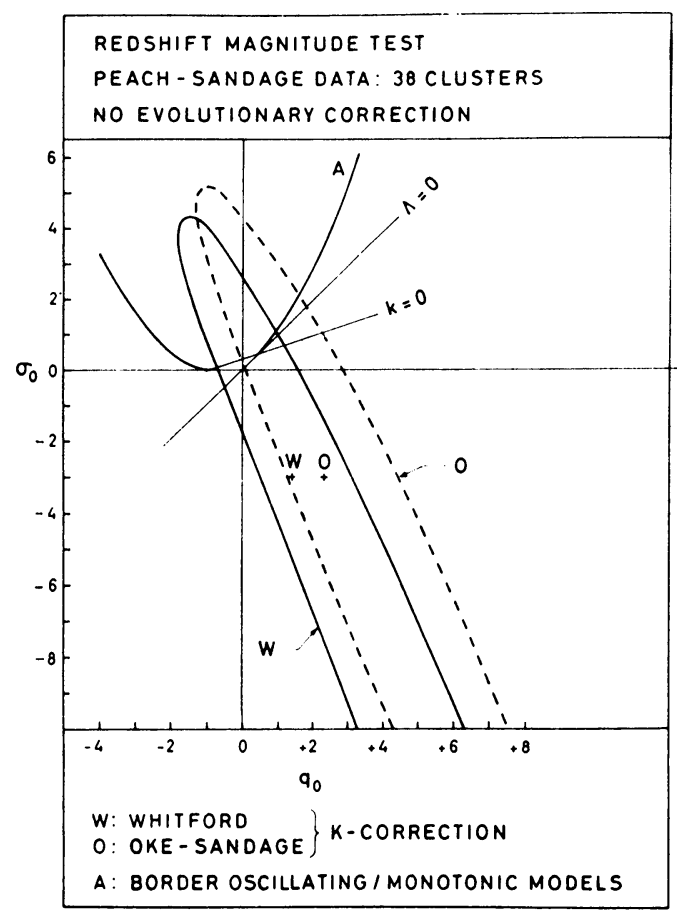

Fig. 1.

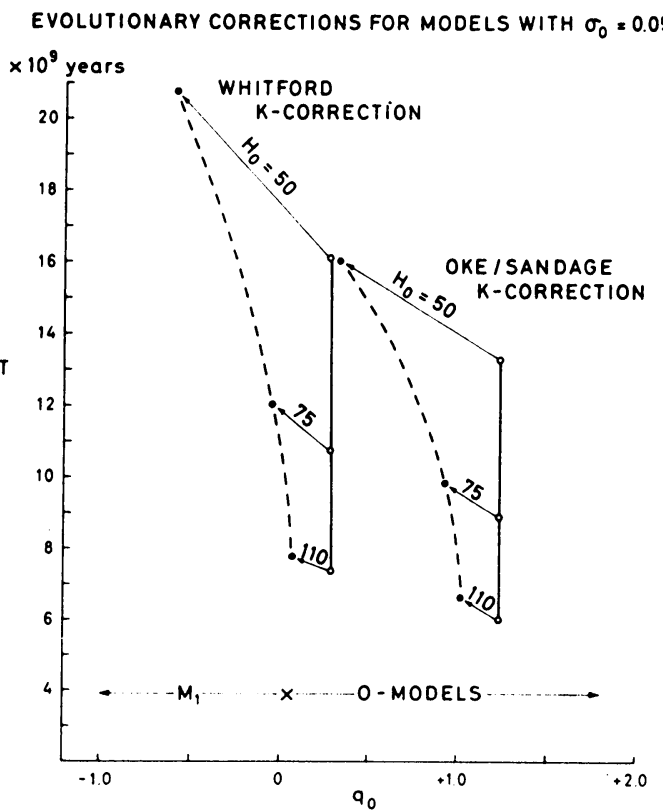

Fig. 2. 
galaxies which may be used to correct this data set. The correction is given by the change in monochromatic (smoothed) magnitude at the wavelength of emission. The size of the correction depends on the value of the Hubble parameter, which provides the time scale. We have used $H=75$ and the two extreme values $H=110$ (van den Bergh, 1970) and 50 (de Vaucouleurs, 1970), to demonstrate the possible effect of evolution.

The result is shown in Figure 2, which gives the change in the best model and the increase in the age of the universe when this evolutionary correction is used. With Whitford's $K$-correction, the best fit is a model with age 12.1 billion years when $H_{0}=75$, and the model type changes from oscillating to monotonic expanding. With the Oke-Sandage $K$-correction, the best models are always of the oscillating type. The actual size of the evolutionary correction is in this case only $-0^{m} .06$ per $10^{9} \mathrm{yr}$.

The model galaxy used for this evolutionary correction has a stellar population like an old galactic cluster, such as NGC 188, with a linear initial main-sequence mass function. The slope of this is such that the resulting present mixture of giants and dwarfs gives good agreement with the available photometry of $g \mathrm{E}$ galaxies (i.e. Johnson's (1966) UBVRIJKL colors, the 15 narrow-band color indices of Wood (1966) and McClure and van den Bergh (1968), and other qualitative indications of contributing stellar types). The luminosity function is not very steep, so in the past the main sequence was fairly well populated above the present turn-off point and the galaxy was then both brighter and bluer.

Figure 3 shows the change of the energy distribution of this model with time:

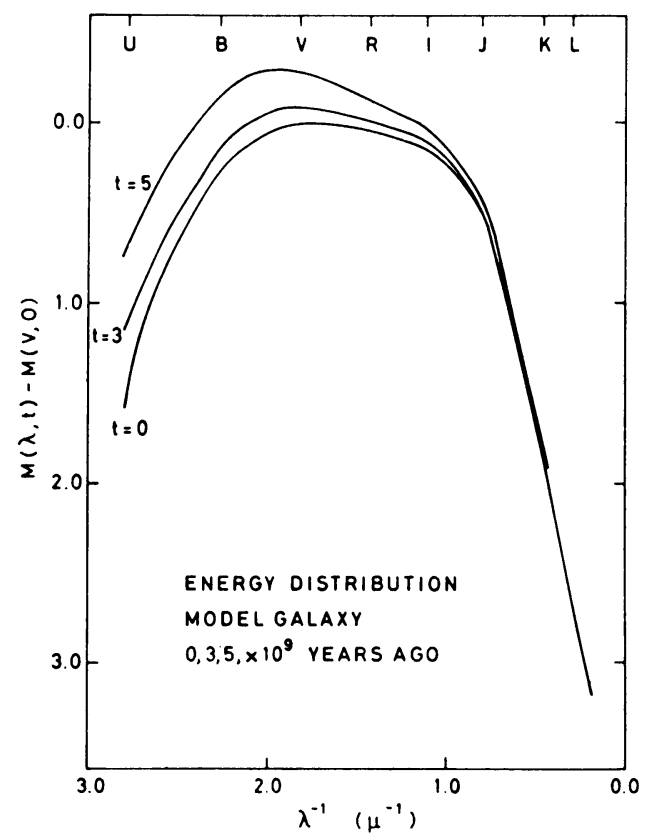

Fig. 3. 
the lowest curve shows the present, the middle curve 3 billion years ago, and the top curve 5 billion years ago. At longer wavelengths, the light is dominated by faint dwarfs, which evolve negligibly. At shorter wavelengths, the light is from giants and the upper main sequence; here evolution is faster because of the earlier main sequence stars in the past. This provides a significant evolutionary correction to observed $V$ magnitudes, because the correction is the change in absolute magnitude at the wavelength of emission, which is shorter than $\lambda_{V}$ by the redshift factor $1+z$.

The evolutionary correction is very uncertain, because the data on $g \mathrm{E}$ galaxies do not determine the slope of the main-sequence luminosity function at all closely. The data can be matched by the shallow slope of this model, and by a model with a steep luminosity function and negligible evolutionary correction (Tinsley, 1970; McClure and van den Bergh, 1968; Chambers and Roeder, 1969).

The results show that evolution could have considerable effect on the determination of a cosmological model from the magnitude-redshift relation, even using this data set that has redshifts up to only 0.2 . We find that quite acceptable values for the age and density of the universe are possible (at the $64 \%$ confidence level) with the correction used here. It is important that the evolutionary correction be found more accurately, for which we require better knowledge of the population of $g \mathrm{E}$ galaxies and a more precise value of the Hubble parameter.

\section{References}

Chambers, R. H. and Roeder, R. C.: 1969, Astrophys. Space Sci. 3, 530.

de Vaucouleurs, G.: 1970, Astrophys. J. 159, 435.

Johnson, H. L.: 1966, Astrophys. J. 143, 187.

McClure, R. D. and van den Bergh, S.: 1968, Astron. J. 73, 313.

Oke, J. B. and Sandage, A. R.: 1968, Astrophys. J. 154, 21.

Peach, J. V.: 1969, Nature 223, 1140.

Peach, J. V.: 1970, Astrophys. J. 159, 753.

Solheim, J.-E.: 1966, Monthly Notices Roy. Astron. Soc. 133, 321.

Stabell, R. and Refsdal, S.: 1966, Monthly Notices Roy. Astron. Soc. 132, 379.

Tinsley, B. M.: 1970, Astrophys. Space Sci. 6, 344.

Van den Bergh, S.: 1970, Nature 225, 503.

Whitford, A. E.: 1971, in Stars and Stellar Systems 9, Ch. 5, (in press).

Wood, D. B.: 1966, Astrophys. J. 145, 36. 